\title{
ENERGY EFFICIENT NETWORK SELECTION USING 802.16G BASED GSM TECHNOLOGY
}

\author{
${ }^{1}$ Anandakumar, H. and ${ }^{2} K$. Umamaheswari \\ ${ }^{1}$ Department of Information Technology, SNS College of Engineering, Coimbatore, India \\ ${ }^{2}$ Department of Information Technology, PSG College of Technology, Coimbatore, India
}

Received 2013-11-05; Revised 2013-11-27; Accepted 2013-12-31

\begin{abstract}
Handover is the mechanism that transfers information from one terminal to another as a user moves through the coverage area of a cellular system. Here the Network selection is the handover decision process between various network environments. This handover decision will be either mobile or network initiated. In 2G GSM handover decision method especially concentrates on Received frequency signals (RFS). Upcoming technologies $(3 \mathrm{G} / 4 \mathrm{G})$ the number of available networks increase the selection process should evaluate additional factors such as cost, network services, network terminology, system conditions, user and operators performances and needed energy to work in an exceedingly network. In Future networks (IEEE or $4 \mathrm{G}$ standards) offer facilities such as network routes, handover routing messages, network and client reports, message exchange with handover triggers and handover negotiation. In this study we have a tendency to discuss the utilization of a cost function to perform associate a network selection exploitation information provided by these standards, such as network coverage or network properties. The efficient function provides flexibility to balance the various factors within the network selection, and our research is focused on rising each seamlessness and energy efficiency of the devices in handovers. We have a tendency to evaluate our approach supported usage scenarios over 2G, 3G and 4G GSM networks. Our results show the optimal networks and handovers were generate appropriate times to increase overall network connectivity as compared to traditional network schemes, at the same time optimizing energy consumption of network devices.
\end{abstract}

Keywords: Handover Management, Energy Efficient Handover, GSM Networks, Optimal Networks, IEEE 802.16g

\section{INTRODUCTION}

In future all mobile devices included with IEEE 802.11/802.16, 3G and 4G networks for wireless communication (Zois et al., 2013). As wireless network access technologies have wide varied characteristics and no single wireless access technology will at the same time offer a low-latency, high-bandwidth and wire-area knowledge service to an outsized variety of mobile users (Liu et al., 2009). The solution is to use a combination of multiple access technologies to provide the best possible connectivity.

A future mobile device will switch between these interfaces depending on its physical environment and requirements (Zois et al., 2013). We refer to this Corresponding Author: Anandakumar, H., Department of Information Technology, SNS College of Engineering, Coimbatore, India

technology, where handover the process of roaming across heterogeneous networks, is one of the most important features. Network selection is a component of the handover procedures to select a network among others networks. Traditionally, network selection has been based on evaluation of the Received Frequency Signals (RFS), e.g., an access point that gives the strongest RFS to the selected nodes (Chuah et al., 2012). In the handover scenario, we need to consider monetary cost, power consumption, network conditions, terminal conditions, user preferences. While significant work has been done on network selection algorithms initiated by the mobile device, the literature survey describing how a mobile device and network operator can cooperate with each other to achieve an optimal network selection in a deployable approach. 
This is an area where standards such as 802.16 or $3 \mathrm{G} / 4 \mathrm{G}$ can facilitate information exchange between these entities to enable the mobile device to obtain relevant network information a priori. In this study we investigate an energy function based network selection architecture assisted by the knowledge of network map coverage.

An energy function based network selection is a flexible algorithm which can take into account the relevant factors to consider with minimal user involvement. For our Literature survey, we used the 802.16e Media Independent Information Service (MIIS) (Geetha and Jayaparvathy, 2011) to provide this information and to the best of our knowledge there has been no work on using 802.16e to optimize network selection in an energy efficient manner using real environmental data (Fang and McNair, 2006).

In this study we proposed a cost based network selection architecture assisted by the knowledge of network map coverage (Zois et al., 2013). A cost based network selection is a flexible algorithm which can take into account the relevant factors to consider with minimal user involvement. For our implementation, we used the 802.16g Independent Information Service (IIS) to provide this information and to the best of our knowledge there has been no work on using 802.16e to optimize network selection in an energy efficient manner using real environmental data. Using this standard we can achieve significant energy efficiency improvement for mobile devices without much overhead. We also proved that by using our algorithm to predict loss of network connectivity, we improve session continuity to support seamless handover.

The rest of the study is organized as follows. In Section II, as the Literature review of this research, our handoff system architecture and technologies are explained. In Section III, the network selection architecture assisted by IEEE $802.16 \mathrm{~g}$ is detailed and evaluated in Section IV, In Section V we discuss our next step where the mobile device handover is assisted by the network and we conclude in Section V.

\section{LITERATURE REVIEW}

We discuss various proposed schemes in this section for improving handover performance using the Independent Information Service (IIS). In conventional handover schemes, L2 triggers are generated after the threshold was reached. The inefficiency was that there were too late or too early triggers (Baliosian and Stadler, 2010).

\subsection{IEEE 802.16e Independent Information Service}

There are currently various efforts underway in standard organizations and industry forums to standardize heterogeneous handovers, including IEEE 802.16e and 3G/4G networks (Houeto and Pierre, 2007). In particular IEEE 802.16e fully compatible with the network services. The media independent handover protocol defines frame formats for exchanging messages between peer IIS Function entities. These messages are based on the layers which are part of Independent Event service, Independent Command service and Independent Information service (Pentikousis, 2010; Buhler and Buhler, 2010).

Our work leverages IIS. The Independent Information Service provides a data store of available networks and network parameters and defines the standard query/response messages to access and retrieve such information for each available access network. Such Information is critical for the handover process. For instance, the information of available networks is very useful for optimizing the network selection process.

\subsection{Simplified Reference Model}

Reference Model probably simplifies the network mobility problem by only depicting the authentication and service authorization servers interfacing to the BS (Base System) over the backbone network. Network layer protocol entities that possible assist mobility over the air interfaces are not depicted it does not describe any specific interface at the data layer for the upper layer control plane or management plane protocols to utilize.

This indication can be generated by an external condition such as signal degradation, by device level condition such as critical battery level requiring a switch to a more energy efficient network, or be user initiated. The step to select on which network to operate, either on a similar network type (homogeneous network) where a link layer transition is sufficient, or on a heterogeneous network where both network and service transitions need to be performed.

Figure 1 shows the Simplified Reference Model of the prototype at the Home network. Applications can use the services provided by the Service station system through the Base station interface. This specification is intended to enhance the radio interface management and control functions and therefore network layer protocols need to an interface to use these data layer protocol definitions. So the protocol architecture defines layers that are exposed to upper layers in a consistent manner for use by control and management plane protocols in a network agnostic topology agnostic manner. 


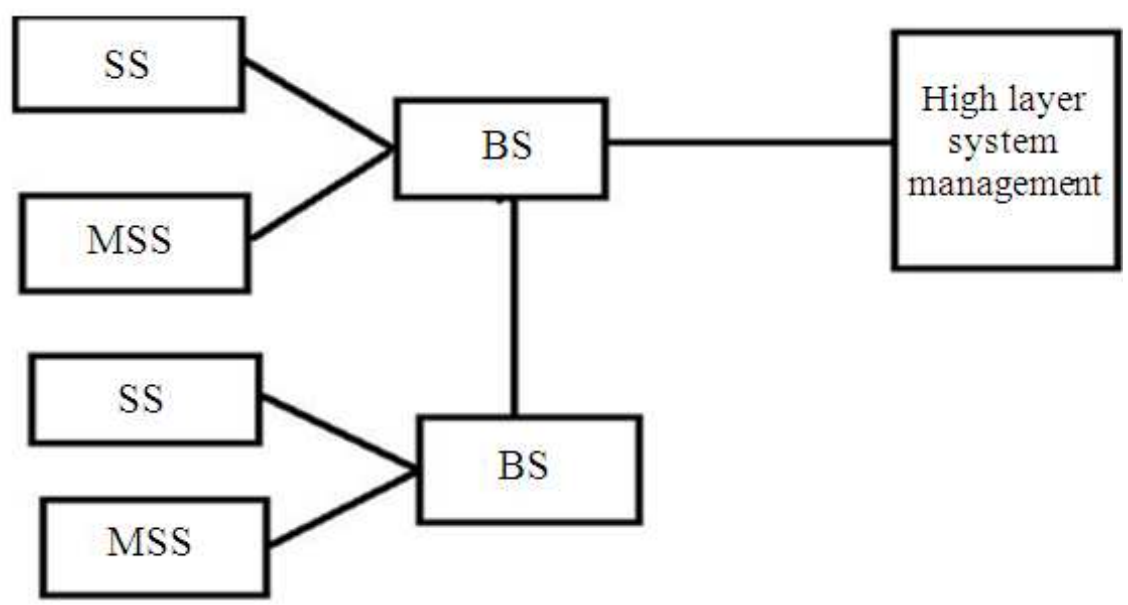

Fig. 1. BS-Base station as defined in 802.16 and adopted by

The services include $802.16 \mathrm{~g}$ Independent Handover (IH) services, connection management and network adaptor management. Applications such as VPN and SIP based applications that are not capable of switching across adaptors can use the connection management service IEEE P802.16g, 2008. Connection managers from other parties can use the $\mathrm{IH}$ and adaptor management services alone and handle connection management themselves.

\subsection{Handover Trigger}

A mobile subscriber (MS) moves around a network and expects to seamlessly maintain connectivity. When a mobile subscriber moves connectivity through one Base Station subscriber (BS) over to another, this is known as a subscriber handover (Liu et al., 2008). The handover procedures and messages that define to support seamless handovers in a wireless network are the focal point of this work.

The accurate and predictive link layer triggers to enable seamless handovers and minimize service interruption. It addresses how to detect link status changes and how to accurately predict such changes. The ability to predict link triggers accurately and earlier in time, especially the Network Line Down and Link Down triggers, will send early warnings to entities such as connection managers and give it extra time to prepare for the handover and seamlessly transfer application sessions (Liu et al., 2008), hence further reduce the service interruption.

The trigger scheme also includes a trigger prediction step. A link trigger is predicted based on predicted future Received Signal Strength Indication values using long and short history windows
(Muscariello et al., 2001). Then a trend analysis algorithm is used to analyze the long and short term trends of Received Signal Strength Indication to prevent false predictions. Evaluation and implementation of the algorithm based on real devices.

\subsection{Cost-Function-Based Independent Service Handover}

A policy-based network selection algorithm with a cost function is first introduced in (AbdelSalam and Olariu, 2011; Liu et al., 2008) and several are proposed in. As described in (Trestian et al., 2013; AbdelSalam and Olariu, 2011). In a handover network with the lowest cost is the network that would provide the most benefit to the user and is the optimal handover target. The increase in value or functionality within a cost-controlled environment is a far more useful than simply paring or lopping elements to a handover.

In the survey the energy functions only consider the traditional network approach such as bandwidth, delay and power by $802.16 \mathrm{e}$, we now have the ability to also consider the physical location of networks, network services, the services offered by these networks, security requirements.

\section{PROPOSED MODEL}

\subsection{IEEE 802.16g Network Selection}

Defined in the original mobile specification, the handover process defines a "backbone" method for requesting mobile subscriber information. When the specification states that the base station may request MS information from a serving BS, however, this does not 
mean that a serving BS should provide it. A base station needs the ability to determine when it should refuse to provide MS information to a requesting $\mathrm{BS}$ The handover description in 802.16e is high level and the $802.16 \mathrm{~g}$ specifies what this request and response (Pahlavan et al., 2000).

Our proposed model of IIS contains two levels of hierarchy. The first level contains High Server (HS) that contains BTS belonging to its specified geographical area. Second level contains Low Server (LS) that contains the information of all of the High level servers and facilitate inter-zone handover. The evaluation parameters are neighbor discovery time and handover delay.

Low information server contains the necessary data of all the available networks belonging to the same operator. When the MN sends a request for the network map to High Level IIS, then that IS sends a reply containing the network map (Schramm et al., 2004). When MN starts moving, it travels to another network, now if the MN sends a request for the network map to High Level IIS, then that IS in turn requests it from the Low Level IIS. The Low Level IIS (LS) returns it to Network Level IIS and the High Level IIS (HS) sends it to the MN.

The architectural model of network selection, which has also been integrated into a system, is shown in Fig. 2. The inputs to the network selection block can be broadly classified as (i) a list of networks (ii) network associated commands and (iii) network and physical associated conditions.

The network selection logic (Chen et al., 2006), considers these inputs in the context of user preferences and policies (Stemm and Katz, 1998), in an ordered list of networks for connectivity. In this study we explore how the layers defined in $802.16 \mathrm{~g}$ can be used by the cost function to select a network in an energy efficient way, the criteria that govern the selection of one network over another in handover policies. The policies include several Parameters like monetary cost, bandwidth, latency, power to which weights can be associated to indicate importance of one parameter over another.

\section{DISCUSSION}

The handover policies can be configured by the user, the first step in network selection is the discovery of networks and a rudimentary way is periodic scanning by all the radios on the device. However, scanning is a power intensive process and network discovery can be made efficient by building a local repository of previously visited and scanned networks. Most modern mobile device support GPS capability, which can be used to store network coordinates.

This handover scheme reduces the periodic scans in previously visited locations, scanning cannot be avoided in new locations. $802.16 \mathrm{~g}$ define an IIS service which is a repository of networks and their static parameters (Wang et al., 1999; Fang and McNair, 2004). The ability of discovering new networks, independent of the connected media, minimizes scanning operation on network interfaces and the time for which interfaces have to remain a power on, therefore prolonging the battery life on the device. Figure 3 compares state machines of the scanning operations, with and without IIS service.

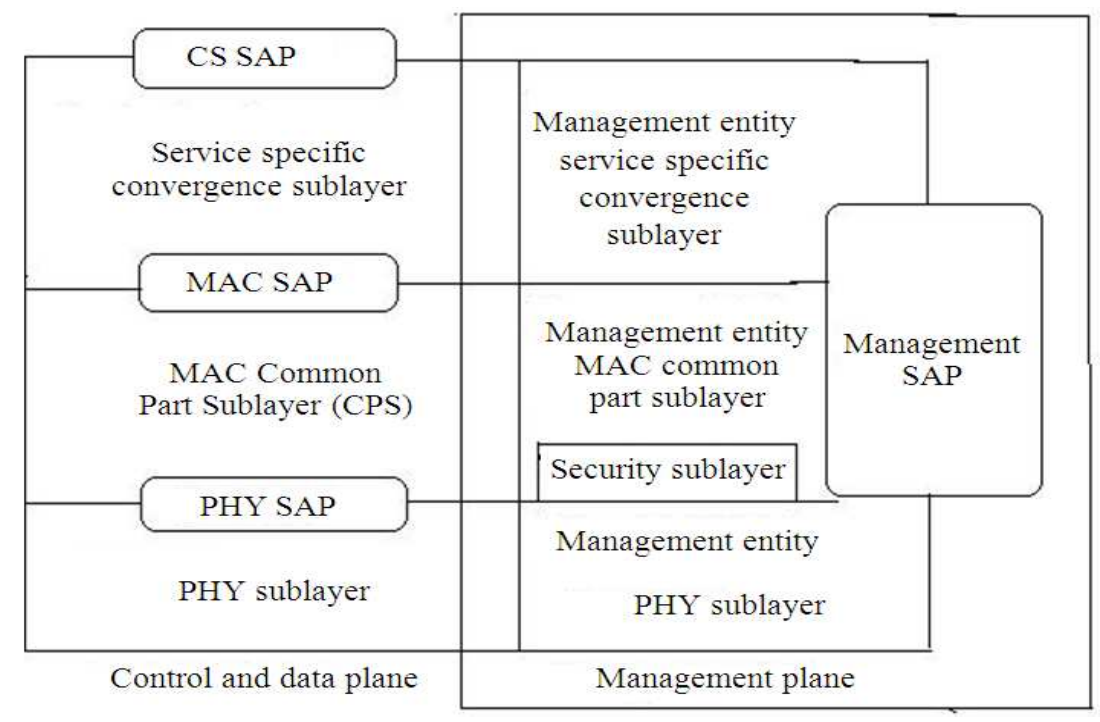

Fig. 2. 802.16g Architectural model 


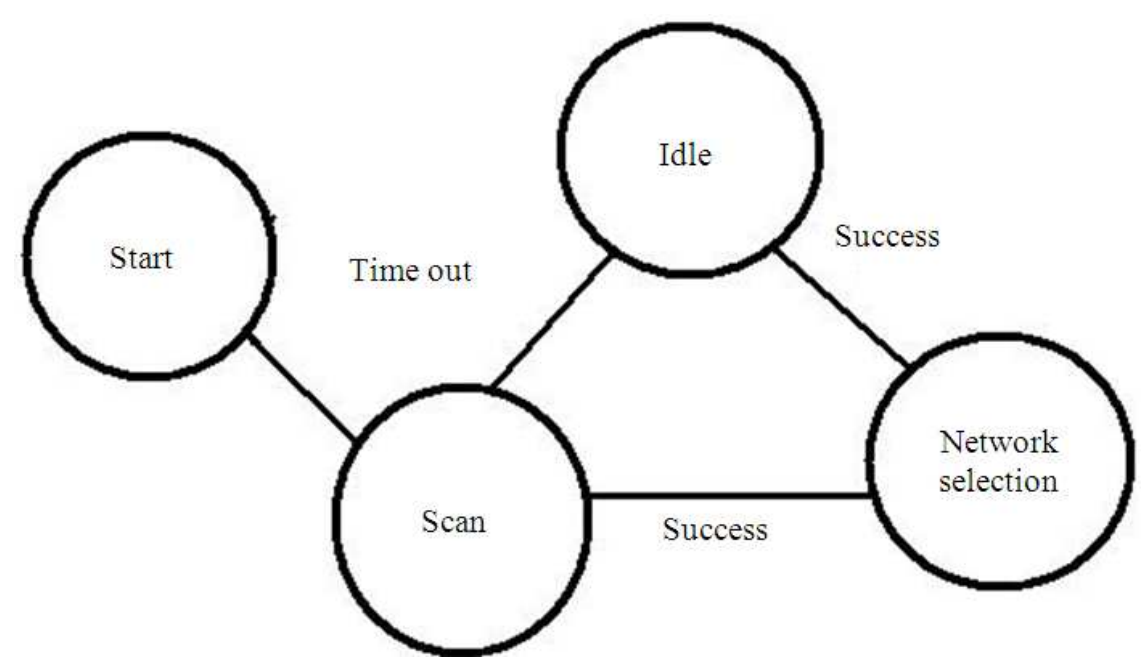

Fig. 3. Scanning and radio on time optimization with out IIS

If the network map from IIS indicates there are no networks in the vicinity the radio need not be turned ON for scanning. If it indicates the presence of a network, the elimination function checks to see if the network's parameters, as provided by IIS service, satisfies all the policies and only then is the network considered as a potential candidate for inclusion in network selection. Thus the information from the IIS along with the elimination function is able to further reduce scanning operations and improve the battery life of the device Fig. 4.

The network selection process is triggered by any of the following events: (i) new networks are discovered, (ii) current network conditions change, (iii) device receive handover commands from the network and (iv) policy changes are initiated by the user or application or operator. The focal point of our research is to obtain network maps from IIS service (Rahmati and Zhang, 2007), detect Network Link down by implementing Media Independent Event Service (MIES) functions and provide IIS information to the cost function to enable energy efficient handover.

\section{EVALUATION}

\subsection{Network Selection}

Our proposed system is to implement a network selection to evaluate different network selection algorithms. The architecture is shown in Fig. 5. The Network consists of three main components.

Network Selector (with cost function), Access Network and Mobile Node. Network Selector implements the network selection algorithm and information used in the algorithm is obtained from the other components. It also implements the function to calculate the cost of each access network regarding the current conditions of the Mobile Node.

Mobile Node provides mobile node conditions such as the name of the current network, status (during communication, scanning, or handover) and static information (number of handover, number of scanning).

Access Network provides conditions of available networks, where signal strength and Access Point (AP) load histories were considered in the simulations. Finally, IIS inputs provide network information that could be obtained from IIS service. For each simulation, we recorded the number of handovers, number of scans and radio duty cycle. We have implemented two trigger methods and two network selection algorithms as listed in Table $\mathbf{1 .}$

Based on the scenario that a user living in a suburban area goes to office in a city area, as shown in Fig. 6, where each circle represents the approximate coverage area of each network traces as the user travels from home to office.

Network 1 is the home $802.16 \mathrm{~g}$ network. Network 2 is an HSDPA based $3 \mathrm{G}$ networks covering the entire route. Networks 3 and 4 are WiMax based on $4 \mathrm{G}$ networks covering the city area. Networks 5,6,7 and 8 are office Wi-Fi networks. The trip from home to office takes $30 \mathrm{~min}$. The RSSI traces for office WiFi networks were collected by walking inside the buildings, using a laptop with a network wireless card. 
Anandakumar, H. and K. Umamaheswari / Journal of Computer Science 10 (5): 745-754, 2014

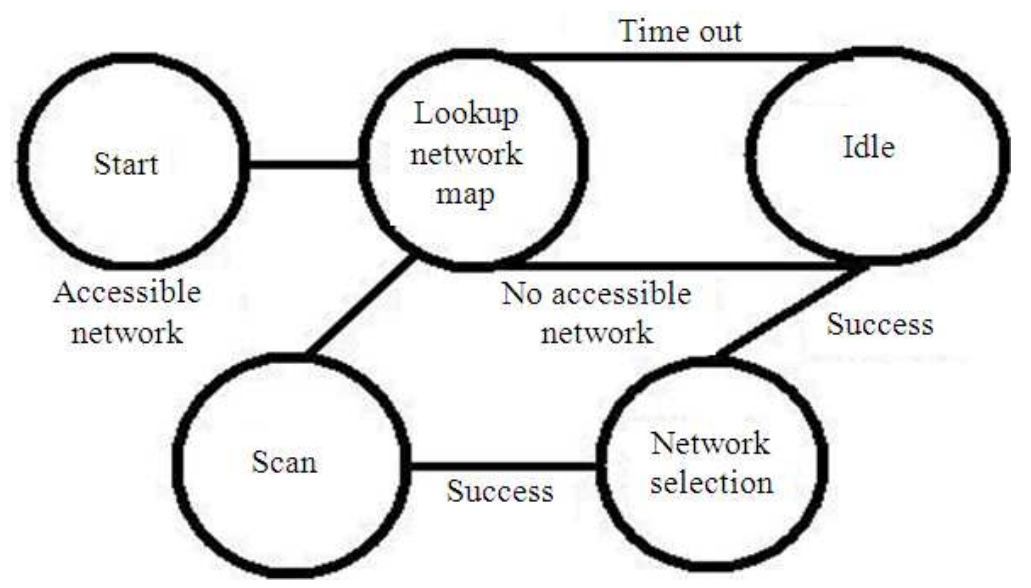

Fig. 4. Scanning and radio on time optimization with IIS

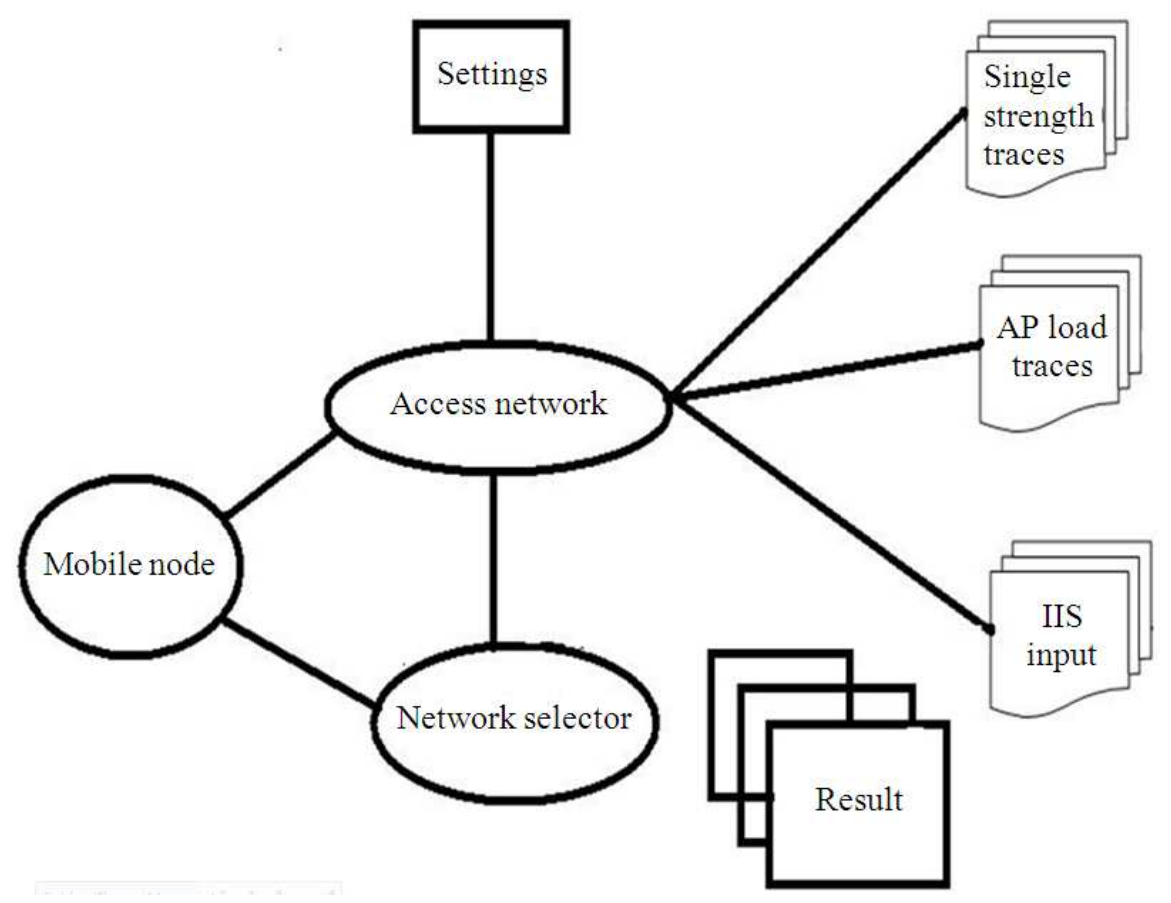

Fig. 5. Network selection

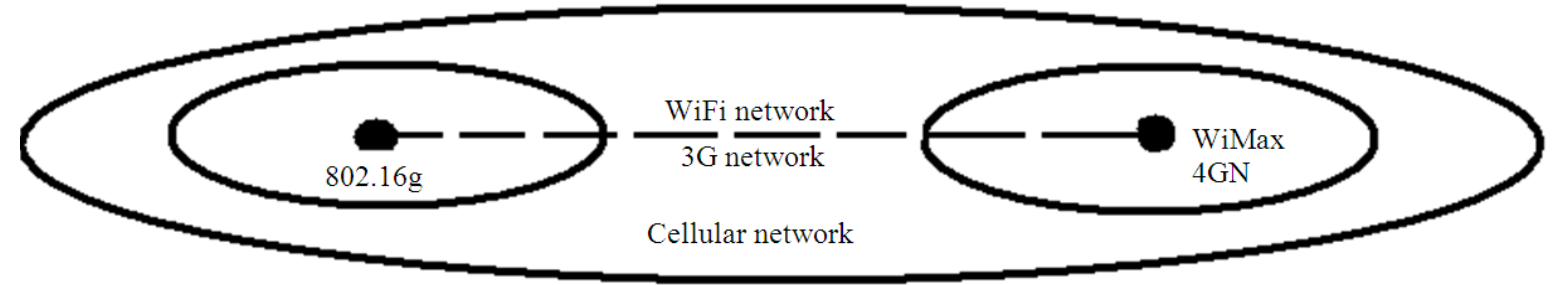

Fig. 6. Network used in RSSI/CINR traces 
Table 1. Implemented features

\begin{tabular}{ll}
\hline Items & Remarks \\
\hline Trigger & Smart trigger (Exponential average with prediction) \\
Network & Static preference $(802.16 \mathrm{~g}>3 \mathrm{G}>4 \mathrm{G})$, energy functions \\
Selection
\end{tabular}

The RSSI traces for the home WiFi network was collected by walking from inside to outside of a house, using the same laptop. The CINR traces for WiMax networks were collected by driving in field trials where WiMax base stations were deployed. Similarly, the 3G (HSPDA) RSSI trace was obtained by driving along a route using a laptop with a Wireless card connecting to the network. We assume the mobile device is a phone type of device and always maintains its connection to cellular/3G networks for phone calls. However, for data transmission, it needs to choose the most appropriate networks, where the network selection is applied.

In our network, AP (or Base Station, BS) load is defined as the total traffic currently served by an AP. The traces of AP loads are generated based on a Markov Modulated Poisson Process (Liu et al., 2008). In practice, AP load can be estimated or obtained in several ways. For example, AP load in WiFi network could be obtained if APs support 802.11 e, or by using methods proposed in (Muscariello et al., 2001). Estimation of cellular or WiMax networks might be done considering the modulation and coding scheme, the received signal level on the broadcast channel, the Carrier-toInterference power ratio $(\mathrm{C} / \mathrm{I})$ and the raw $\mathrm{BER}$ estimate (Chen et al., 2006). The cost function used in the experiments is as follows:

$$
\mathrm{C}=\mathrm{WB} \ln \frac{1}{\mathrm{~B}}(+) \mathrm{Wp} 1 \mathrm{np}+\mathrm{Wc} 1 \mathrm{nC}
$$

Where:

$\mathrm{B}=$ Denotes available bandwidth

$\mathrm{P}=$ Denotes power

$\mathrm{C}=$ Denotes the monetary cost

On our network, to get the available bandwidth of an AP or BS, we subtracted the AP's load from the highest data rate that can be provided by the network (this information could be provided by IIS service). For WiFi networks, we assume that they are $802.16 \mathrm{~g}$ APs with a 11 Mbps data rate; for WiMax, we assume $30 \mathrm{Mbps}$ as the highest available data rate (the data rate depends on the modulation scheme, the coding scheme, (GPP, 2008) and for $3 \mathrm{G}$, we assume $1.2 \mathrm{Mbps}$ data rate. Effectively estimating the available bandwidth is an active research area and is beyond the scope of this study. For monetary cost, we assume that $\mathrm{WiFi}$ is of flat cost (fixed monthly fee), while costs for WiMax and $3 \mathrm{G}$ access are based on the amount of bytes transmitted through that network. We assume that WiMax cost somewhat less than $3 \mathrm{G}$.

For power consumption, studies have shown that $3 \mathrm{G}$ card IEEE P802.21/D14, 2008 consumes more transmit power than the WiFi card (Schramm et al., 2004), partly due to the long radio range. We assume that WiMax card consumes higher power than a WiFi card in transmission for the same reason but less than $3 \mathrm{G}$ card.

\subsection{Performance Analysis}

Our ultimate goals for network selection and seamless handover are to select the most appropriate network, optimize for energy efficiency (Ahmavaara et al., 2003; Toh, 2001) and switch at the best timing. In this section, we evaluate our algorithms in each aspect of the goals.

\subsection{Network Selection using Cost Function}

To study the effectiveness and flexibility of cost function based network selection, we run experiments with different weights for each factor in the cost function.

In both experiments, the weight of power is assigned 0.9 . The weight of bandwidth was 0.1 , the weight of monetary cost was 0.1 . Hence, in both experiments, power is the utmost factor in selecting an access network, WiMax was selected in the first experiment, while WiFi was selected in the second experiment (recall that both are more power more efficient than $3 \mathrm{G}$ ). The results demonstrate the flexibility of the cost function to take different factors into consideration.

\subsection{Energy Efficient Network Selection with IIS}

802.16g IIS support can both reduce handover delay (by reducing the time for network discoveries) and reduce power consumption during network selection. In this section, we study the power saving benefit by applying IIS (Min and Chandrakasan, 2003). As explained before, the operation in the network selection process that consumes the most power is scanning for available networks and obtaining the necessary information.

Typically, without IIS optimization, to discover and obtain network information in time, WiFi networks are scanned once minute and cellular networks are scanned every $30 \mathrm{sec}$ to $1 \mathrm{~min}$ until a connection is established. Our measurements indicated that when WiFi radio scans every minute, on average mobile devices (e.g., an Ultra Mobile PC) power consumption increased by about $100 \mathrm{mw}$ over time, if the 
WiFi radio goes to low power after each scanning. When cellular radio scans every minute, the device's power consumption increased 200 to $600 \mathrm{mw}$ (power consumption varies with different radio chipsets). For WiMax, our measurements indicate 100 to $300 \mathrm{mw}$ increase of platform power. Hence, a significant amount of power would be saved by disabling scanning or even turning off the radio, if it is known that a radio is out of the coverage of accessible.

In section 3, we explained how IIS could help save energy of a mobile device. To evaluate the energy savings enabled by applying IIS service, we assume that for each radio, the scanning power is the same when it is connected to a network or not. Also, since we assume that $3 \mathrm{G}$ is always one (Kulik et al., 1999). This might not always be the case in real implementations.

Once a device is connected, scanning process to update network information can be provided by current network and hence could cost less power than when the radio is not connected. Note that in this case, majority of scanning power is consumed when a radio is available in the simulation setup and the mobile device maintains the $3 \mathrm{G}$ network connection all the time, we only compare the scanning power consumptions for WiFi and WiMax radio below. As shown in Fig. 7a, without IIS service, the radio has to remain on and scan for networks all the time (although it may go to low power state after each scan), in order to obtain information for network selection.

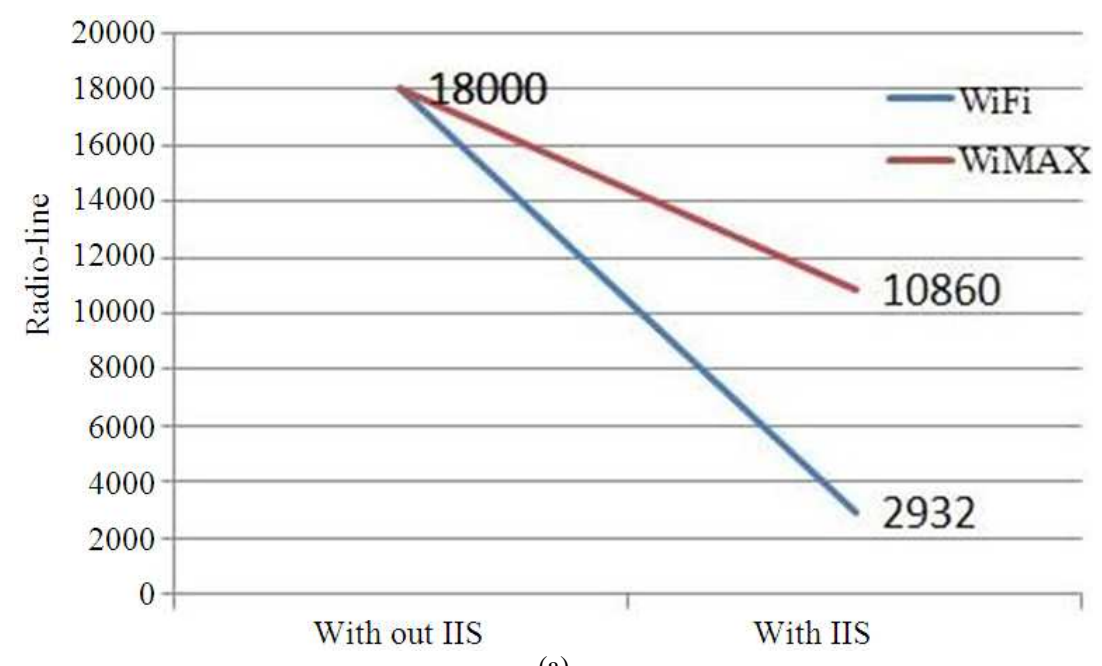

(a)

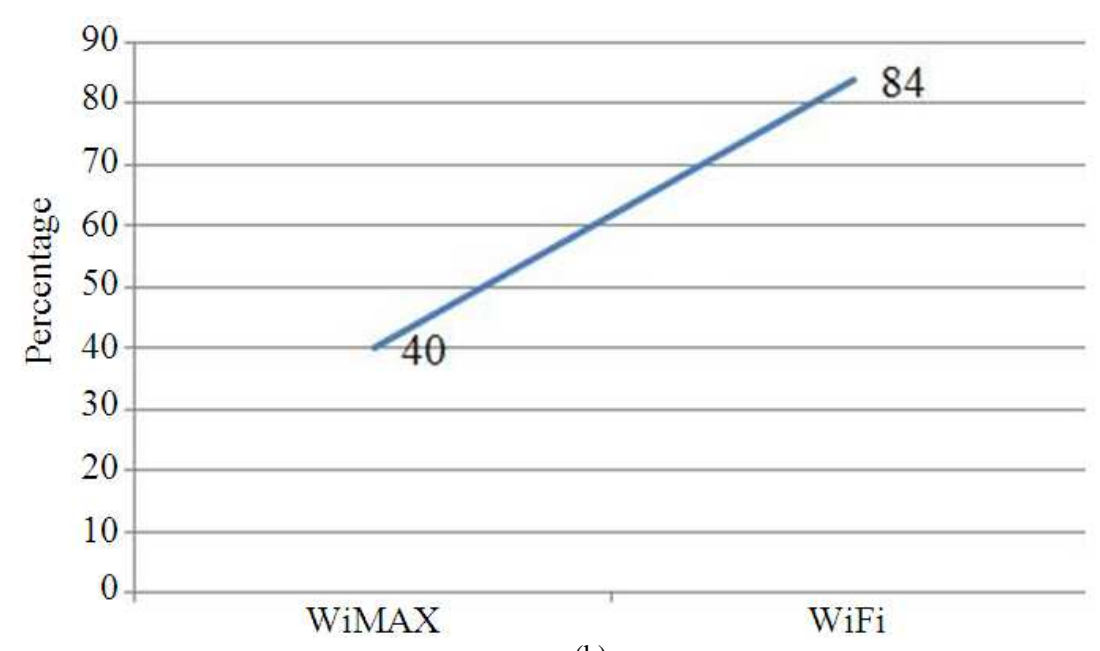

(b)

Fig. 7. (a) Power saving benefits of applying IIS (b) Overall analysis of power saving benefits 
However, with IIS service, when a radio is out of coverage, there is no need (Intanagonwiwat et al., 2000) to turn it on for scanning during that period. Hence the radio-on time is greatly reduced especially when networks are far less than $100 \%$ of coverage. Figure $\mathbf{7 b}$ shows that by turning off a radio, the proportion of scanning power has been saved. For WiMax, since $40 \%$ of time the mobile device was out of coverage, at least $40 \%$ of scanning power could be saved and for WiFi, $86 \%$ of scanning power could be saved. Therefore, IIS optimization is especially useful when there is a significant portion of time that a device would be out of coverage of some networks. Networks coverage varies a lot depending on where people live, their daily commute pattern and so on. A recent study (Schramm et al., 2004; Stoica et al., 2002), shows that by collecting traces from different people's daily life, on average, $51 \%$ of a person's daily life are within WiFi coverage.

In the future, it is still unlikely that $\mathrm{WiFi}$ coverage will be $100 \%$. WiMax and $3 \mathrm{G}$ have better coverage (Fu et al., 2003; De Couto et al., 2003) however, they are much less than $100 \%$ of coverage today and will likely remain under $100 \%$ at least in the near future. Therefore, by applying IIS optimization, a significant amount of power could be saved by avoiding unnecessary scanning activities.

\section{CONCLUSION}

Review of the main research activities which show the importance of independent information service to make the handover performance optimized in wireless devices, the overall energy consumption of the device is a key issue, when the number of frequencies on these devices increases and at the same time end-users expect a seamless connectivity across all frequencies. To address these issues we proposed flexible network selection architecture to optimize seamless handover operations as well as to optimize the energy efficiency of these devices.

First, as a component of this architecture handover triggers improves the seamless aspect by providing early indication of connectivity and optimizing the endusers' connected time.

Second, we proposed to enrich a mechanism of cost function based network selection by using additional properties of the mobile devices and network services such as $802.16 \mathrm{~g}$ Information Service, to optimize the network selection process for optimal energy efficiency.

Third, Energy saving is in $802.16 \mathrm{~g}$ Information Service provides information enabling the device to reduce its scanning frequency and obtain available networks with limited energy overhead. We also discuss that there is an almost negligible energy cost to acquire the required additional information from the $802.16 \mathrm{~g}$ Information Server using an appropriate request rate. As a next step we're now integrating our energy efficiency network system assisted handover mechanism to enhance seamless handovers.

\section{REFERENCES}

AbdelSalam, H.S. and S. Olariu, 2011. Toward efficient task management in wireless sensor networks. IEEE Trans. Comput., 60: 1638-1651. DOI: 10.1109/TC.2010.264

Ahmavaara, K., H. Haverinen and R. Pichna, 2003. Interworking architecture between $3 \mathrm{gpp}$ and wlan systems. IEEE Commun. Mag., 41: 74-81. DOI: 10.1109/MCOM.2003.1244926

Baliosian, J. and R. Stadler, 2010. Distributed autoconfiguration of neighboring cell graphs in radio access networks. IEEE Trans. Netw. Ser. Mana., 7: 145-157. DOI: 10.1109/TNSM.2010.1009.I9P0330

Buhler, J. and G. Buhler, 2010. Traffic-aware optimization of heterogeneous access management. IEEE Trans. Commun., 58: 1737-1747. DOI: 10.1109/TCOMM.2010.06.090182

Chen, J.C., T.C. Chen, T. Zhang, E. V.D. Berg, 2006. Effective AP selection and load balancing in IEEE 802.11 wireless LANs. Proceedings of the IEEE Global Telecommunications Conference, Nov. 27Dec. 1, IEEE Xplore Press, pp: 1-6. DOI: 10.1109/GLOCOM.2006.724

Chuah, S.P., Z. Chen and Y.P. Tan, 2012. Energyefficient resource allocation and scheduling for multicast of scalable video over wireless networks. IEEE Trans. Multimedia, 14: 1324-1336. DOI: 10.1109/TMM.2012.2193560

De Couto, D.S.J., D. Aguayo, J. Bicket and R. Morris, 2003. A high-throughput path metric for multi-hop wireless routing. Proceedings of the 9th Annual International Conference on Mobile Computing and Networking, Sept. 14-19, ACM Press, New York, USA., pp: 134-146. DOI: 10.1145/938985.939000

Fang, Z. and J. McNair, 2004. Optimizations for vertical handoff decision algorithms. Proceedings of the IEEE Wireless Communications and Networking Conference, Mar. 21-25, IEEE Xplore Press, pp: 867-872. DOI: 10.1109/WCNC.2004.1311300

Fang, Z. and J. McNair, 2006. Multiservice vertical handoff decision algorithms. Eur. J. Wireless Commun. Netw., 2006: 52-64. DOI: $10.1155 / \mathrm{WCN} / 2006 / 25861$ 
Fu, Z., P. Zerfos, H. Luo, S. Lu and L. Zhang et al., 2003. The impact of multihop wireless channel on tcp throughput and loss. Proceedins of the IEEE Societies 22nd Annual Joint Conference of the IEEE Computer and Communications. Mar. 30-Apr. 3, IEEE Xplore Press, pp: 1744-1753. DOI: 10.1109/INFCOM.2003.1209197

Geetha, S. and R. Jayaparvathy, 2011. Dynamic bandwidth allocation for multiple traffic classes in IEEE 802.16e WiMax networks: A petrinet approach. J. Comput. Sci., 7: 1717-1723. DOI: $10.3844 /$ jcssp.2011.1717.1723

GPP, 2008. 3 Architecture enhancements for non-3GPP accesses (3GPP TS 23.402).

Houeto, F. and S. Pierre, 2007. Jitter characterization in admission control and pricing issues in integrated multiservice networks. J. Comput. Sci., 3: 965-979. DOI: 10.3844 jessp.2007.965.979

Intanagonwiwat, C., R. Govindan and D. Estrin, 2000. Directed diffusion: A scalable and robust communication paradigm for sensor networks. Proceedings of the 6th annual international conference on Mobile computing and networking, Aug. 06-11, ACM Press, New York, USA., pp: 56-67. DOI: $10.1145 / 345910.345920$

Kulik, J., W. Rabiner and H. Balakrishnan, 1999. Adaptive protocols for information dissemination in wireless sensor networks. Proceedings of the 5th Annual ACM/IEEE International Conference on Mobile Computing and Networking, Aug. 15-19, ACM Press, Seattle, WA., pp: 174-85. 10.1145/313451.313529

Liu, H., C. Maciocco and V. Kesavan, 2009. Energy efficient network selection and seamless handovers in mixed networks. Proceedings of the IEEE International Symposium World Wireless, Mobile and Multimedia Networks and Workshops, Jun. 1519, IEEE Xplore Press, Kos, pp: 1-9. DOI: 10.1109/WOWMOM.2009.5282451

Liu, H., C. Maciocco, V.S. Kesasvan and A. Low, 2008. A smart triggering scheme to reduce service interruption during heterogeneous handovers. Proceedings of the IEEE International Conference on Dependable Systems Networks, Jun. 24-27, IEEE Xplore Press, Anchorage, AK, pp: 430-439. DOI: 10.1109/DSN.2008.4630113

Min, R. and A. Chandrakasan, 2003. Top five myths about the energy consumption of wireless communication. Proceedings of the ACM SIGMOBILE Mobile Computing and Communications Review, Jan. 1-1, ACM Press, New York, USA., pp: 65-67. DOI: $10.1145 / 881978.881998$
Muscariello, L., M. Mellia, M. Meo, R. Lo Cigno, M. Ajmone Marsan, 2001. A simple markovian approach to model internet traffic at edge routers. Dipartimento di Elettronica.

Pahlavan, K., P. Krishnamurthy, A. Hatami, M. Ylianttila and J.P. Makela et al., 2000. Handoff in hybrid mobile data networks. Personal Commun., 7: 34-47. DOI: 10.1109/98.839330

Pentikousis, K., 2010. In search of energy-efficient mobile networking. IEEE Commun. Mag., 48: 95103. DOI: 10.1109/MCOM.2010.5394036

Rahmati, A. and L. Zhang, 2007. Context-for-Wireless: Context-sensitive energy-efficient wireless data transfer. Proceedings of the 5th International Conference On Mobile Systems, Applications and Services, Jun. 11-14, ACM Press, New York, pp: 165-178. DOI: 10.1145/1247660.1247681

Schramm, P., F. Peter and H.G. Muller, 2004. Olofsson, Cell selection in mobile radio systems. US Patent 6542742 IEEE STD 802: 16-2004, Table B.29.

Stemm, M. and R.H. Katz, 1998. Vertical handoffs in wireless overlay networks. Mobile Netw. Applic., 3: 335-350. DOI: 10.1023/A:1019197320544

Stoica, I., D. Adkins, S. Zhuang, S. Shenker and S. Surana, 2002. Internet indirection infrastructure. IEEE/ACM Trans. Netw., 12: 205-218. DOI: 10.1109/TNET.2004.826279

Toh, C.K., 2001. Maximum battery life routing to support ubiquitous mobile computing in wireless ad hoc networks. IEEE Commun. Mag., 39: 138-147. DOI: $10.1109 / 35.925682$

Trestian, R., O. Ormond And G.M. Muntean, 2013. Energy-quality-cost tradeoff in a multimedia-based heterogeneous wireless network environment. IEEE Trans. Broadcast., 59: 340-357. DOI: 10.1109/TBC.2013.2244790

Wang, H.J., R.H. Katz and J. Giese, 1999. Policyenabled handoffs across heterogeneous wireless networks. Proceedings of the 2nd Workshop on Mobile Computing Systems and Applications, Feb. 25-26, IEEE Xplore Press, New Orleans, LA., pp: 51-60. DOI: 10.1109/MCSA.1999.749277

Zois, D.S., M. Levorato and U. Mitra, 2013. Energyefficient, heterogeneous sensor selection for physical activity detection in wireless body area networks. IEEE Trans. Signal Process., 61: 15811594. DOI: 10.1109/TSP.2012.2236320 